\title{
Recent Experimental Results Claim for a Rigorous Understanding of Nuclear Forces
}

\section{Petrus HR dos Anjos*}

Departamento de Física, CAC-UFG, Catalão, Go, Brazil

In this brief note, we discuss why some recent experimental results in particle physics highlight the need for a more thorough and rigorous understanding of Quantum Chromodynamics.

Even after the many years since Quantum Chromodynamics (QCD) was proposed as the model for strong interactions, we (un)fortunately do not have satisfactory answers to some questions that arises in particle physics. Perhaps the most fundamental problem in quantum physics is to determine the existence and the spectral properties of any quantum field theory. In QCD, it is essential to establish on a rigorous basis the low energy-momentum (E-M) spectrum of particles and their bound states. In particular, one needs to prove the existence of multiquark states, i.e. mesons, baryons and their bound states. Consequently, it is also a fundamental step towards a more complete understanding of the Yukawa (nuclear) force from first principles.

The need of a more accurate understanding of the strong force was recently highlighted by the discovery of some exotic hadrons in particle accelerators around the world. Let consider for instance the case of four quark (anti-quark) states, called tetraquarks. These states have been observed in many experiments such as the BELLE (2008) and LCHb (2014) collaborations and now there is an unambiguous experimental proof of their existence [1]. The embarrassing fact that tetraquarks have been discovered experimentally and were not predicted is an indication of our poor understanding of QCD. Furthermore, the true nature of the tetraquark incited a fierce debate among particle physicists about the correct picture of matter at the quantum scale [2]. Most believed tetraquarks were a some kind of hadronic molecule (essentially, two standard mesons orbiting each other, each one made of one regular quark and one antiquark) while a smaller group saw them as particles in which the two quarks and two antiquarks overlapped in a small volume of space. The same unclear scenario holds concerning other possible composite particles as e.g. glueballs, tetraneutrons and other particle molecules. In particular, these experimental results claim for a more complete (,less controversial!,) and mathematically rigorous understanding of the QCD, that can help to bridge the gap between QCD and the effective baryon, effective meson picture of nuclear forces through single and multiple boson exchange arising from the Yukawa interaction [3].

Some progress in this direction has been reached considering QCD on the lattice (much less progress was made in the continuum). One approach is to consider an imaginary-time functional integral formulation of $3+1$ lattice QCD with Wilson's action. This approach was employed in [4] for a 2- flavors (global symmetry $\mathrm{SU}(2)_{f}$ ) and 3-colors (local gauge symmetry $\mathrm{SU}(3)_{c}$ ) QCD model (the same scheme can be employed to analyze a QCD model with more avors). At strong coupling, a polymer expansion or other cluster methods ensure that in this model the thermodynamic limit of correlations exists and truncated correlations have exponential tree decay. Also, the limiting correlations are translational invariant and extend to analytic functions in the hopping parameter $(0<$ kappa $<<1$

) and in the pure-gauge coupling $(0<\beta \ll \kappa)$. Associated with this model, there is an underlying Hilbert space $\mathrm{H}$, constructed through a correspondence between expectations and inner products in $\mathrm{H}$ by a

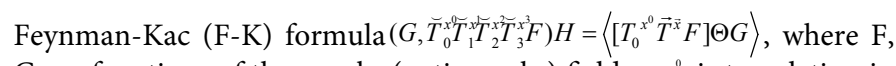
$\mathrm{G}$ are functions of the quarks (anti-quarks) fields, $T^{x^{0}}$ is translation in time by $x^{0}>0, \vec{T}^{\vec{x}}=T_{1}^{x^{1}} T_{2}^{x^{2}} T_{3}^{x^{3}}$ is translation in space by $\vec{x}=\left(x^{1}, x^{2}, x^{3}\right)$ , the symbol ${ }^{\vee}$ denotes operators in $H$ and $\Theta$ is an anti-linear operator which involves time rejection. By taking the Fourier transform of the F-K formula, a connection is established between the joint spectrum of the self-adjoint E-M operators $P^{i=1,2,3}$ and $P^{i=1,2,3}$ (where $\widetilde{T}_{0}=e^{-H}$ and $T_{-} j=e^{\wedge}\left\{\mathrm{iP}^{\wedge} \mathrm{j}\right\}$ ), and singularities of correlations in complex momentum. Letting $\varepsilon\left(\lambda^{0}, \vec{\lambda}\right)=E_{0}\left(\lambda^{0}\right) \Pi_{1}^{3} F_{j}\left(\lambda^{j}\right)$ denote the product of the spectral families of $\breve{T}_{0}, \breve{T}^{1}, \breve{T}^{2}$ and $\breve{T}^{3}$, by the spectral theorem, we have the spectral representations $\breve{T}_{0}=\int_{-1}^{1} \lambda^{0} d E_{0}\left(\lambda^{0}\right), \breve{T}_{j=1,2,3}=\int_{-\pi}^{\pi} e^{i \lambda j} d F_{j}\left(\lambda^{j}\right)$. The spectral representations allow one to make a rigorous correspondence between singularities of the Fourier transform of correlations and the E-M spectrum. Note that by only detecting the exponential decay rate of the correlations does not establish a relationship with particles. Particles correspond to isolated dispersion curves in the E-M spectrum and decay rates can be related with other spectral points, if any. For instance, we can be dealing with a spectral point in the middle of a spectral band or, when narrowly split states are present, we can detect an energy value in between two energy levels.

In the strong coupling regime, the above method can be used to rigorously validate the eightfold way picture for baryons (with asymptotic mass $-3 \ln \kappa$ ) and mesons (with asymptotic mass $-2 \ln \kappa$ ), by obtaining each isolated dispersion curves in the E-M spectrum. The hadron masses were given by convergent expansions in $\kappa$ and $\beta$, this analysis include the hadron mass splittings. The method can also be used to search for bound states. This is done using a ladder approximation of a lattice Bethe-Salpeter (B-S) equation $D=D_{0}+D_{0} K D$, where the kernel $\mathrm{K}$ describes the interaction between the two particles. This approach can be understood by an analogy with Schrdinger operators in $\ell^{2}\left(\mathbb{Z}^{d}\right)$ Consider the resolvent $(H-z)^{-1}=\left(H_{0}-z\right)^{-1} V\left(H_{0}-z\right)^{-1}$ where $H_{0} \approx \frac{\kappa}{2} \Delta,(-\Delta$ is the lattice Laplacian $)$ and the interaction potential is $\mathrm{V}=\mathrm{L}+{ }^{2} \mathrm{~W}$, where $\mathrm{L}$ correspond to a short distance (dominant) component and $\mathrm{W}$ also involves long distances, but has exponential decay. Taking into account only the $\mathrm{L}$ contributions, the resolvent equation can be solved explicitly and the spectral results follows. In this ladder approximation of the B-S equation, some two-baryon and two-meson states can be detected, including a total spin-one, total isospin zero two-baryon state like the deuteron. Also, in this scenario

${ }^{*}$ Corresponding author: Petrus HR dos Anjos. Departamento de Física, CACUFG, Catalão, Go, Brazil, Tel: 202- 5564-3441-5322; E-mail: petrus@ufg.br

Received October 2, 2014; Accepted October 14, 2014; Published November 5, 2014

Citation: Anjos PHR (2014) Recent Experimental Results Claim for a Rigorous Understanding of Nuclear Forces. J Phys Math 5: 126 doi: 10.41722090-0902.1000126

Copyright: (c 2014 Anjos PHR. This is an open-access article distributed under the terms of the Creative Commons Attribution License, which permits unrestricted use, distribution, and reproduction in any medium, provided the original author and source are credited. 
Citation: Anjos PHR (2014) Recent Experimental Results Claim for a Rigorous Understanding of Nuclear Forces. J Phys Math 5: 126 doi: 10.41722090-0902.1000126

Page 2 of 2

the calculations show an absence of baryon-meson bound states, which indicate the nonexistence of pentaquarks, as suggested by recent the experiments.

While these scheme can be regarded as limited and far from physical reality, it worth to point that this scheme gives us conditions for an explicit analytic treatment of the low-lying E-M spectrum of the QCD, and so far, it point to a possible way for a rigorous validation of some important spectral results. Also, we note that this method incorporates the ingredients to extend the bound state results beyond the ladder approximation [5]. On another hand, the results obtained by this approach (so far) appear to suggest that the binding between hadrons becomes more unlike to happen in more complex cases. In this way, there is a real need to obtain rigorous results in a more realistic scenario. Together with a better understanding, from first principles, of the full features of the interaction holding the quark components together inside observed hadrons and nuclei, these are presently some of the very challenging open questions in $\mathrm{QCD} /$ particle physics and therefore deserves better attention.

\section{References}

1. Aaij R (2014) LHCb Collaboration, Phys Rev Lett.

2. Maiani L, Piccinini F, Polosa AL, Riquer V (2014) Z(4430) and a new paradigm for spin interactions in tetraquarks. Phys Rev $D$.

3. Machleidt R (2001) The nuclear force in the third millennium. Nucl Phys A 689 : $1-2$

4. Faria da Veiga PA, O'Carroll M, Neto AF,Dos Anjos PHR (2011) On the Absence of Pentaquark States from Dynamics in Strongly Coupled Lattice QCD. Int J Mod Phys A 26.

5. Schor R, O'Carroll M (2000) Decay of the Bethe-Salpeter Kernel and Bound States for Lattice Classical Ferromagnetic Spin Systems at High Temperature. J Stat Phys 99: 1265-1279. 